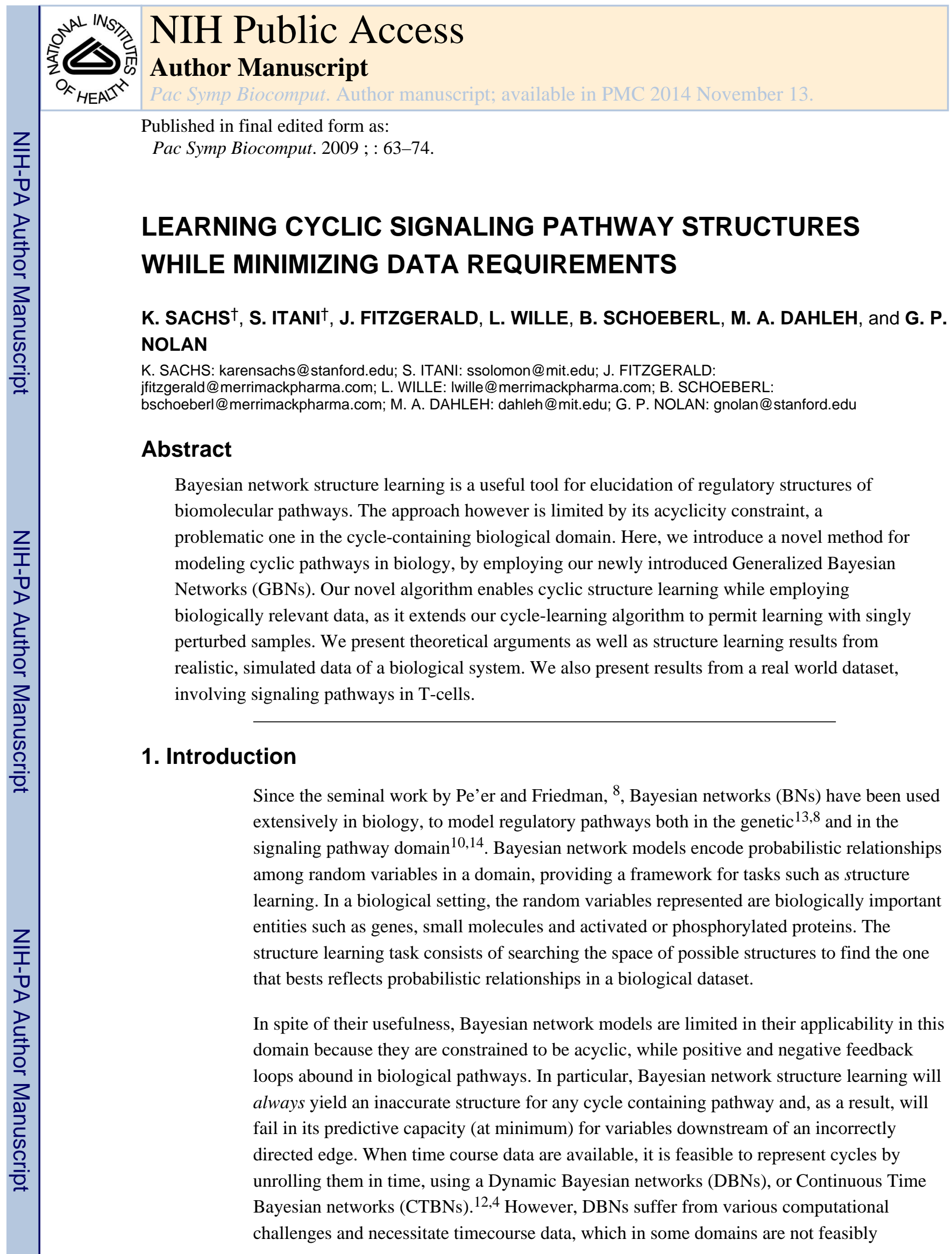

\footnotetext{
$\dagger$ These authors contributed equally to this work.
} 
attainable in an applicable form (e.g. $\left.{ }^{10}\right)$. Therefore, it would be useful to find an approach for learning cyclic structures from static 'snapshot' data, collected at a single timepoint from a dynamic system.

We have recently developed a formalism for representing cyclic structures using Generalized Bayesian networks (GBNs), a form of Bayesian networks that we have generalized to encompass cycles. ${ }^{1}$ This formalism enables structure learning in a cyclic domain, relying on perturbations which break the cyclic structure. Far from requiring an exhaustive set of perturbations, the algorithm is designed to minimize the number of interventions needed, requiring as few as merely one intervention per cycle for accurate structure learning.

Here, we present the first ever application of GBNs to biological signaling pathways. We apply the algorithm to realistic, biologically relevant data from a differential equation model of IGF signaling. Next, we substantially modify the structure learning algorithm to bring it incrementally closer to applicability in a biological domain, by minimizing the algorithm's data requirements. We then test this new algorithm on a reduced set of the synthetic data and compare its results. Finally, we perform structure learning on real data in which T-cell signaling molecules were measured using multidimensional flow cytometry from ${ }^{10}$, and demonstrate that our new algorithm is able to elucidate cyclic structures in signaling pathways.

\section{Background and Methods}

We present background on BNs and GBNs, as well as the synthetic data used in this study.

\subsection{Bayesian networks}

Bayesian networks ${ }^{15}$, represent probabilistic dependence relationships among multiple interacting components, illustrating the effects of pathway components upon each other in the form of an influence diagram- a graph $(G)$, and a joint probability distribution. In the graph, the nodes represent variables (the biomolecules) and the (lack of) edges represent (conditional in)dependencies ${ }^{15}$. For each variable, a conditional probability distribution $(C P D)$ quantitatively describes the form and magnitude of its dependence on its parent(s). Due to the factorization of the joint probability distribution, the graph must be acyclic, meaning that it must not be possible to follow a path from any node back to itself. ${ }^{15}$

\subsection{Generalized Bayesian Networks}

When building models of pathways, Bayesian network models have a number of strong advantages. They are flexible and interpretable, they can handle interactions of arbitrary complexity (given sufficient data), and they can smoothly incorporate both prior knowledge and interventional data in a principled way. However, they have one serious drawback for modeling biological systems: they are unable, as described above, to handle cycles in a static model. Because cycles abound in biological pathways, a static Bayesian network model usually cannot hope to capture all influence connections. 
To address this problem and enable the use of Bayesian network models in a cyclic domain, we recently introduced Generalized Bayesian Networks (GBNs), a generalization of Bayesian networks to the cyclic domain. ${ }^{1}$ In ${ }^{1}$ we also present an algorithm in which the GBN formalism is used to recover causal structure given interventional (static) data, in acyclic or cyclic domains. The algorithm is briefly presented below.

GBN structure learning-Call the set of variables $V$ and the subset of variables with interventions available $I$. Note that the inhibitors are activity inhibitors, typically small molecule inhibitors which perturb the activity- rather than the abundance- of a protein.

\section{Algorithm: Learn Causal GBN structure}

0: Start with a Causal GBN and an intervention set $I$.

1: [Probing experiments] Collect sets of i.i.d. samples under no-intervention and single-intervention data, i.e. when node $i$ is intervened at, for each $i$ in $I$.

2: Call subroutine 'detect descendants' to recover descendant information for all nodes in I. Based upon response of variables to perturbations, further explained in subroutine below.

3: Identify cycles. Based upon perturbations which affect the abundance of the target variable. Because the inhibitors affect protein activity, an effect on abundance must be due to a loop from the target back to itself.

4: Do Bayesian Network learning with the cycles broken (by interventions on nodes we call "cycle breakers"- a set of variables that include at least one representative from each cycle) and integrating the descendant information.

5: Determine the correct edges needed to close the cycles, by detecting the children of the cycle breakers.

6: Recover all missing edges in the DAG, and complete the Directed Cyclic Graph structure of the Causal GBN.

The subroutine used in step 2 is an important subroutine that uses the sensitivity of descendants to perturbations on their ancestors. This sensitivity can be described as the assumption that if a distribution of the parents of a variable change, then the distribution of the variable itself will change too. This idea was introduced in the context of GBNs ${ }^{1}$. To test whether a node was affected by a perturbation on another node (and thus deduce the anscestor/descendant relation), the following subroutine is used:

\section{Subroutine: Detect descendants}

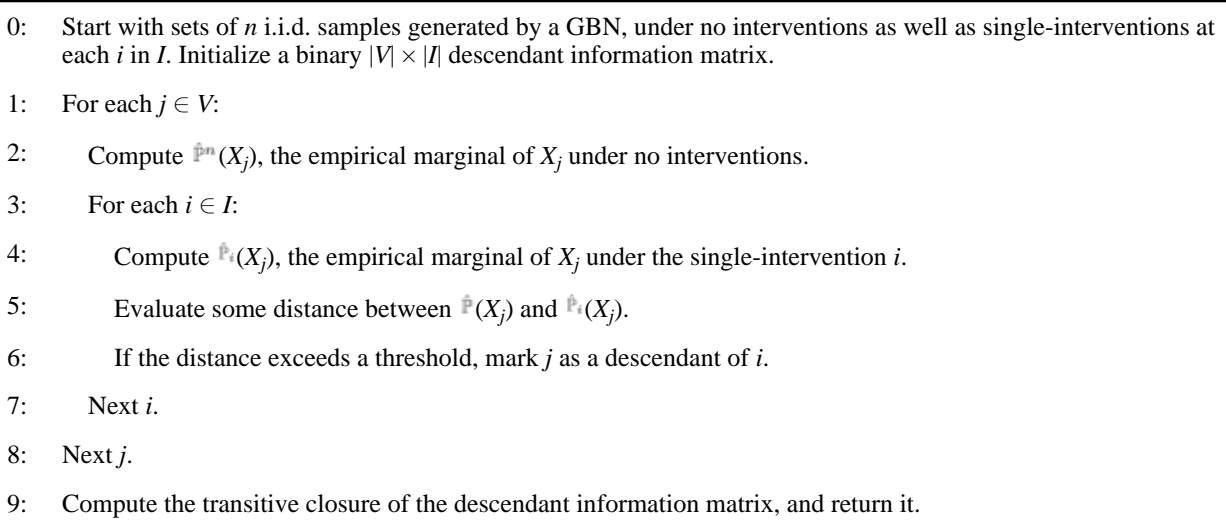

The details of this algorithm are presented in ${ }^{1}$ and it is proven that given enough data and interventions, this algorithm is guaranteed to recover the causal structure of the data. Note 
that step 4 removes all cycles to enable structure learning using standard BN. The algorithm developed in this work avoids this step, to avoid the requirement for conditions involving inhibitor combinations. As such, this modified algorithm is far better suited to the logistic reality of limited experimental data.

\subsection{Synthetic Data and Model of IGF Signaling}

To produce synthetic data, we use a mass action kinetic model describing the dynamics of the Insulin-like growth factor (IGF) signaling pathway. ${ }^{5,6}$ IGF signaling is important in normal cell physiology, as well as pathological states such as cancer. A schematic representation is shown below. Mass action kinetic equations were used to create the model in MATLAB Sim-Biology v2.1.

There are three directed cycles in the model: IRS $\rightarrow$ PI3K $\rightarrow$ AKT $\rightarrow$ mTOR $\rightarrow$ IRS, GRB2/SOS $\rightarrow$ RasGTP $\rightarrow$ MEK $\rightarrow$ ERKp $\rightarrow$ ERK $\rightarrow$ GRB2/SOS, and GRB2/SOS $\rightarrow$ RasGTP $\rightarrow$ MEK $\rightarrow$ Erk $\rightarrow$ GRB2/SOS.

The stimulus employed is IGF, in addition, up to five perturbations are employed, at IGFR, MEK, PI3K, AKT, and mTOR, corresponding to actual existing small molecule inhibitors. All of the perturbations are activity inhibitions, that is, they inhibit the protein's activity, not permitting the targeted protein to phosphorylate other proteins. We generated measurements from four different time points, under 17 total conditions composed of IGF stimulus plus various combinations of inhibitors. For each condition, 1000 unique, randomly selected initial conditions (i.e. molecule concentrations) were employed- the equivalent of collecting 1000 unique cells in a flow cytometry experiment, or performing western blots on 1000 samples. Simulated "measurement noise" was also added.

The model was created by Jonathan Fitzgerald and colleagues at Merrimack Pharmaceuticals. It is a highly accurate imitation of the true biological system ${ }^{19}$, and, accordingly, provides us with synthetic but realistic data, similar to the data one might acquire from a high throughput measurement technology (as in ${ }^{10}$ ). It is a flexible and realistic source of 'true to life' synthetic data, which, because it has a known ground truth model, provides an invaluable tool for assessing success of structure learning efforts.

\section{Algorithm Description}

In this section, we detail an algorithm for structure learning of cyclic networks with single perturbation data. This algorithm depends on first detecting the cycles in the network using perturbations, then using the data where each cycle is broken to recover its structure.

Algorithm: Learn Structure

\footnotetext{
0: $\quad$ Start with a set of variables $V$ and a set of single-intervention variables $I \subset V$.

1: [Experiments] Collect sets of i.i.d. samples under no-intervention and single-intervention data, i.e. when node $i$ is intervened at, for each $i$ in $I$.

2: $\quad$ Call subroutine 'detect descendants' to recover descendant information for all nodes in $I$.

3: Identify one node per cycle from $I$, and the set of such nodes $I_{\mathcal{C}}$. Nodes in $I_{\mathcal{C}}$ are detected as self-descendants.
} 


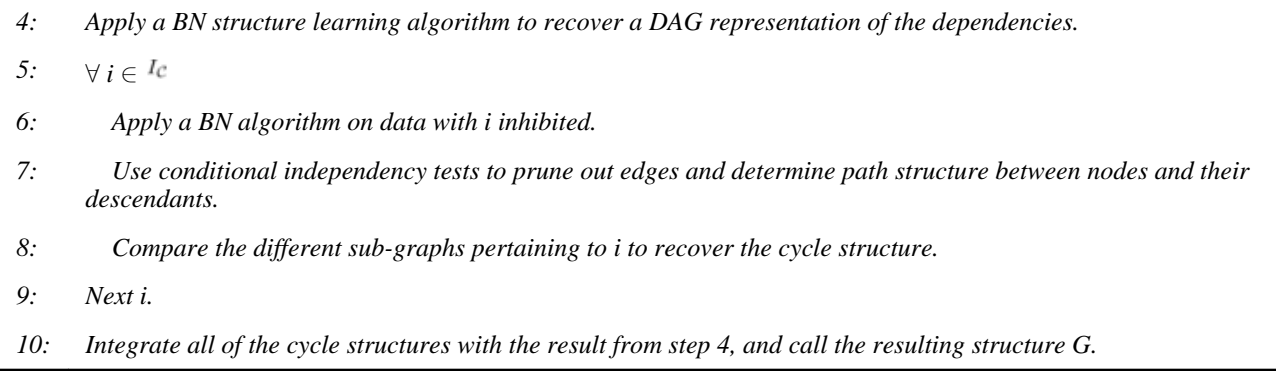

\section{Algorithm Analysis}

In this section we aim to study the algorithm we presented is Section 3, mainly focusing on the reasons it can be expected to have good performance as well as where it is expected to have limitations.

The main assumption needed for the algorithm to recover the true structure is that the BN structure learning algorithm recovers the correct structure of the non-cyclic part of the graph whenever it is applied. This means that the existence of a cycle shouldn't interfere with the conditional independencies between variables outside the cycle itself.

From the study in ${ }^{18}$, it can be seen that the cycle does not usually affect the rest of the graph dramatically. This is mainly because the structure of the d-separation in a cycle (what dependencies/independencies conditioning on every set of variables induce) is the same as that of a loop with the same structure of the cycle except for one reversed edge. Thus the BN learning algorithm would tend to recover the whole structure with some reversed or missing edges from the cycle, and usually nodes that are outside the cycle will not be affected.

We therefore expect this algorithm to perform well, even compared to algorithms that use multiple-perturbation data. This is mainly because this algorithm reinforces and corrects the structure recovered by the $\mathrm{BN}$ algorithm. It does so using the descendant information from the perturbation analysis and the conditional independency analysis (step 7).

\section{Results}

In this section, we present structure learning results for BNs and GBNs, as well as the new GBN algorithm presented above. BN learning is implemented as in ${ }^{10}$. Data was discretized to 4 levels using interval discretization. We show the results obtained from the IGF-model based data with combinations of inhibitors, 17 conditions total, followed by conditions employing only single perturbations (6 conditions total), up to 1000 datapoints were used per condition. Lastly, we show results using a flow cytometry dataset of T-cell signaling molecules. The models presented are averaged over 20 individual results, edges with confidence $>0.8$ are included. In the following graphs a dotted edge is an incorrect edge that was predicted, a black edge is a correct edge. For the GBN based algorithms, the degree of shifting for which a variable is considered to be a child of the perturbed variable is a model parameter. Here, we use a $20 \%$ shift as the cutoff, chosen based on an observed bimodality 
among the candidate children. Another good approach would be to determine the cutoff by randomizing the data and determining the magnitude of a null shift.

\subsection{Multiple perturbations}

The original GBN structure learning algorithm requires conditions in which multiple perturbations are applied simultaneously. Results from the BNs and the original GBN structure learning algorithm are shown below, both use 17 different conditions, each with different combinations of the 5 inhibitors. The BN results, shown on the left, find most of the model edges (missing the connection between mTor and IRS, as well as Ras/Raf and Mek, and shifting the Erk $\rightarrow$ Grb2Sos connection to Erkp), however, the model contains 10 additional, noncausal edges. The GBN results improve on the BN results substantially, missing only the Erk $\rightarrow$ Grb2Sos edge, which it too shifts to Erkp, and including only 2 extra noncausal edges.

What causes the extraneous edges? We were originally surprised to see them, as the data comes from a clean, well defined system which, we had assumed, would be free of confounding elements that usually induce noncausal edges. Upon a closer look, we uncovered two likely culprits. One is the dynamics of the system, something we do not explicitly contend with in single timepoint, 'static' models, but which can have confounding effects induced by the history of a molecule being best represented by a different molecule, an effect called entanglement (see ${ }^{7}$ for a discussion of this topic). A second is technically a causal effect, though it does not result from enzymatic alteration of one molecule by another. This is an effect that may be present when multiple molecules interact with the same intermediate molecule. Consider for instance the edge between IGFR and Pi3k, an extraneous, noncausal edge. Both IGFR and Pi3k interact with IRS, an interaction that takes IRS out of the pool of available, free IRS. Thus, if IGFR binds an IRS molecule, the IRS is no longer available to bind Pi3k (though the total amount of active IRS is unaffected, since IRS can be active while bound to its up or downstream partners). Though IGFR is not causally affected by IRS, it is nevertheless competing with Pi3k for IRS binding. IGFR binding affects the effective amount of available IRS that Pi3k "sees", inducing a dependence between IGFR and Pi3k that is independent of IRS abundance. We call this effect occupancy, because it is the result of limited available occupancy on the intermediate molecule-if the upstream molecule is bound, the downstream one cannot bind, and vice versa. The impact of occupancy in terms of whether an edge is likely to appear probably depends on the specifics of the interactions. for example, the duration of binding of each molecule, and the extent to which the intermediate molecule is present in excess or is in short supply.

\subsection{Single perturbations}

Above, we formulate a novel algorithm also based on GBNs, but able to perform structure learning from data with just one perturbation per sample. We present BN and GBN results below, both use data from 6 total conditions. The BN result includes many fewer edges than the one found with 17 conditions, possibly because the total number of datapoints is smaller (the BN scoring scheme penalizes complexity, so some edges appear only if sufficient data is available. Though we did not enforce that an equal number of datapoints be used 
regardless of the number of conditions, this would be a useful idea to try). The BN result contains 7 extra edges, fails to orient some connections, and misses three connections (missed connections not shown). The GBN model contains one extra edge, misses two connections and shifts the Erk $\rightarrow$ Grb2Sos edge to Erkp as before. We note that it is not clear to us why the edge between Erk and Grb2Sos is so commonly shifted to Erkp. Occupancy does not seem to be a factor, because binding times are short and Erk is not in short supply. It may be an effect of the dynamics of the system as discussed in ${ }^{7}$. Regarding data requirements, we use here all available single perturbation conditions, a total of 5 perturbational and 1 observational conditions. However, the learning results are robust down to just two conditions, albeit with a loss of some edges (results not shown). Note however that the descendent information from all 5 perturbations is employed. This points to the possibility that a low throughput approach (such as western blots) could be used to detect descendants, and a high throughput approach (such as flow cytometry) employed for just a small set of perturbation conditions. This approach may save resources and reduce expense while still yielding good results.

\subsection{Single perturbations with real data}

To test our algorithm on real data, we employ a real-life dataset created using multidimensional flow cytometry, described in ${ }^{10}$. Measurements of T-cell signaling proteins are reported under observational as well as perturbational conditions with just one perturbation per condition. Perturbation conditions were available for Mek and Akt. The represented pathway is thought to contain (at least) two cycles: $R a f \rightarrow M e k \rightarrow p 44 / 42$ (Erk) $\rightarrow A k t \rightarrow R a f$, and $R a f \rightarrow M e k \rightarrow p 44 / 42 \rightarrow$ Raf ${ }^{10}$. We focus on the proteins involved in these two cycles. BN and GBN results are shown below, with BN results on the left and GBN on the right. The BN result misses one edge and fails to orient two edges. The GBN result is nearly perfect, but it does fail to orient one edge.

\section{Conclusions}

In this paper, we demonstrate the first-ever application of cyclic-structure learning in signaling pathways using both synthetic and real life data, with score-based Bayesian networks. We present a novel structure learning algorithm grounded in the GBN formalism, and capable of handling single perturbations conditions, to reduce the algorithm's data demands. We test the original GBN algorithm on synthetic data from an accurate, differential equation model of IGF signaling. We then test the novel extended GBN algorithm formulated here, on both synthetic and real life data. In each case, our algorithm demonstrates clearly superior performance, in terms of elucidation of cyclic structures, correctly orienting model edges and even elimination of extraneous edges.

In our exploration using synthetic data, we discover unexpected edges and propose two main reason for their appearance- confounding effects of the dynamics of the system (discussed in a companion paper, ${ }^{7}$ ), and occupancy effects, based on multiple molecules binding to the same intermediate molecule, thus creating competition-like effects, even though not all of them may be causally affected by the intermediate molecule. This latter concept needs a more rigorous treatment, a topic that we will explore in future work. We also had available multiple timepoints from the synthetic data. For this study, a timepoint was selected 
arbitrarily, but the effect of timepoint selection will be discussed in ${ }^{7}$, using the same synthetic dataset, along with the original GBN algorithm.

In the biological domain, we are often interested in a causal model, partly for the insight and understanding such a model conveys with respect to the modeled system, and partly for the possibility for system predictions which it enables. In disease states for instance, a characterization of the altered biological network can serve to guide therapeutic interventions. A truly causal model which includes correctly oriented edges is crucial- with it, a useful target can be identified and potentially detrimental effects can be avoided. Whereas previous attempts at modeling biological pathways with Bayesian networks have yielded useful results, the prevalence of cycles have confounded those efforts, compromising the causal nature of the learned models. With this work, by overcoming the acyclicity constraint, we have brought the structure learning capability incrementally closer to learning truly causal models.

\section{Acknowledgments}

This work was supported by a Leukemia and Lymphoma Society post doctoral fellowship to K.S., and NIH grants N01-HV-28183, U19 AI057229, 2 P01 AI36535, U19 AI062623, R01-AI065824, 1 P50 CA114747, 2P01 CA034233-22A1, and HHSN272200700038C, NCI grant U54 RFA-CA-05-024 and LLS grant 7017-6 to G.P.N.

\section{References}

1. Itani S, Ohannessian M, Sachs K, Nolan GP, Dahleh MA. NIPS. 2008 submitted.

2. Calder M, Vyshemirsky V, Gilbert D, Orton R. 2006

3. Nodelman U, Shelton C, Koller D. UAI. 2002

4. Nodelman U, Shelton C, Koller D. UAI. 2003

5. Carlson CJ. Biochem Biophys Res Comm. 2004; 316(2):533-9. [PubMed: 15020250]

6. Moelling K, et al. J Biol Chem. 2002; 277(34):31099-106. [PubMed: 12048182]

7. Itani S, Sachs K, Fitzgerald J, Wille L, Schoeberl B, Nolan G, Dahleh M. in preparation.

8. Friedman N, Linial M, Nachman I, Pe'er D. J Comput Biol. 2000; 7:3-4.

9. Friedman N. Science. 2004; 303:5659.

10. Sachs K, Perez O, Pe'er D, Lauffenburger DA, Nolan GP. Science. 2005

11. Sachs K, Gifford D, Jakkola T, Sorger P, Lauffenburger DA. Science STKE. 2002

12. Friedman, N.; Murphy, K.; Russell, S. Proceedings of the Fifteenth Annual Conference on Uncertainty in Artificial Intelligence; 1999. p. 139-147.

13. Hartemink AJ, Gifford DK, Jaakkola TS, Young RA. Pac Symp Biocomput. 2001

14. Woolf PJ, Prudhomme W, Daheron L, Daley G, Lauffenburger QDA. Bioinformatics. 2004

15. Pearl, J. Probabilistic Reasoning in Intelligent Systems. Morgan Kauffman; 1988.

16. Pearl J, Verma TS. Second KR. 1991:441-452.

17. Heckerman, D.; Meek, C.; Cooper, GF. Computation, Causation, and Discovery. Glymour, C.; Cooper, GF., editors. MIT Press; 1999. p. 141-166.

18. Richardson TS. UAI. 1996:454-461.

19. Schoeberl B, Fitzgerald JB, Wille L, West K, Pace E, Harms B, Gibbons F, Donis E, Grantcharova V, Kumar A, Kudla A, Nielsen UB. Understanding IGF signaling dynamics through computational modeling. in preparation. 


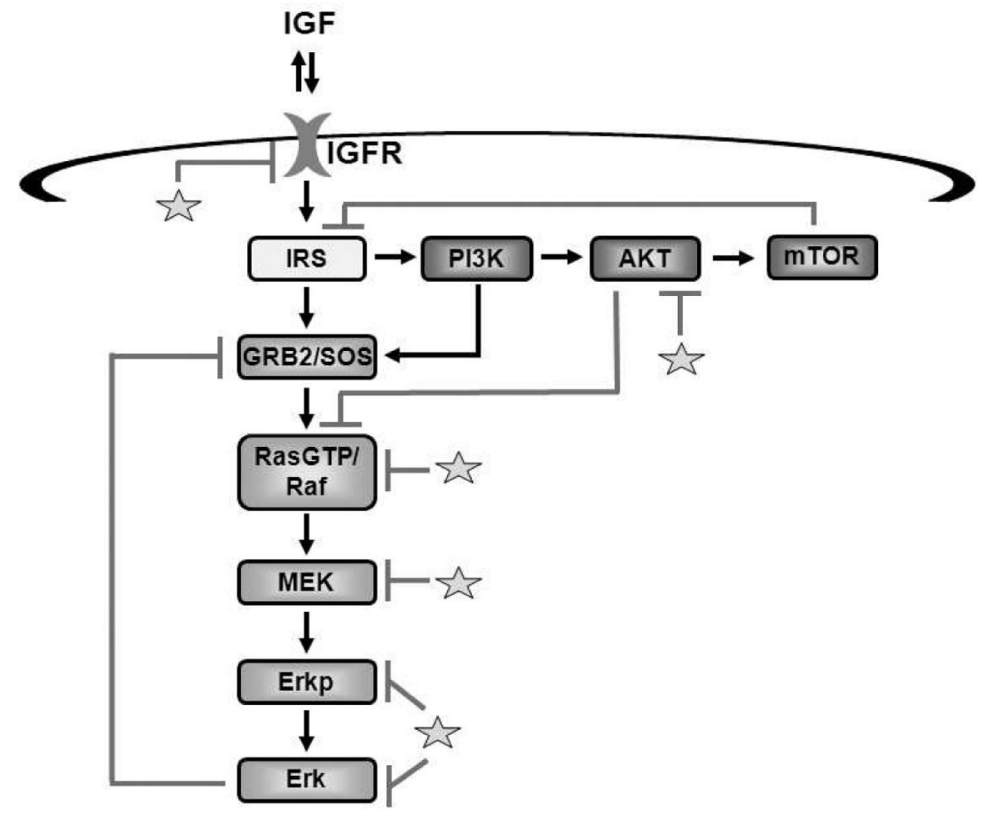

Figure 1. True structure of the underlying dynamic system in IGF signaling Each node represents the active "on state" of the protein. Stars indicate phosphatases acting on the target molecules. Perturbations in the form of small molecule inhibitors are available for Mek, Akt, Pi3k, IGFR and mTor. The simulated data mimics these inhibitors by blocking enzyme activity. 


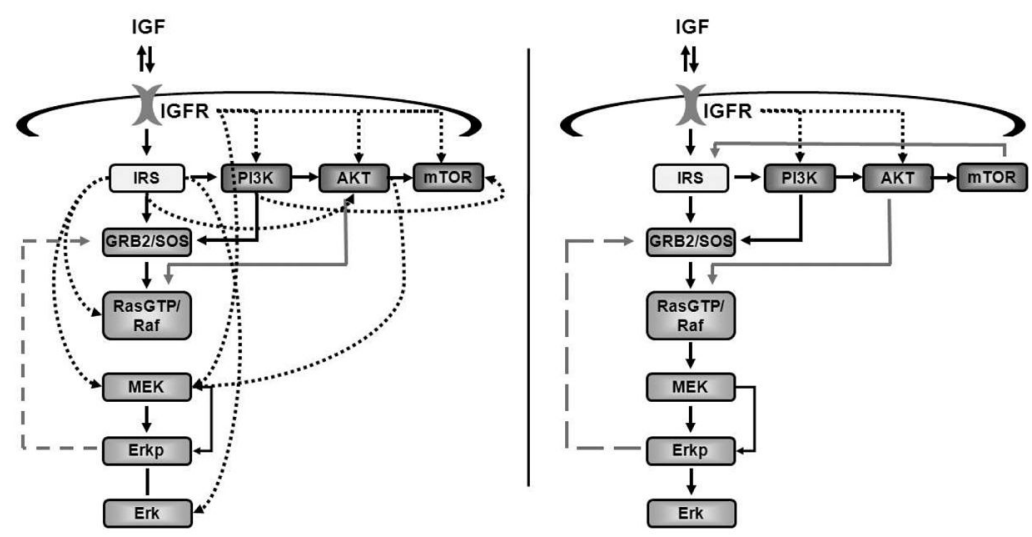

Figure 2. BN and GBN results from synthetic data, using multiple perturbations per sample $\mathrm{BN}$ results are shown on the left, GBN results on the right. Broken arrows are noncausal. Missed edges are not indicated; the BN model misses 2 edges and shifts the Erk $\rightarrow$ Grb2Sos edge to Erkp, the GBN model misses no edges but shifts the Erk $\rightarrow$ Grb2Sos edge as well. 


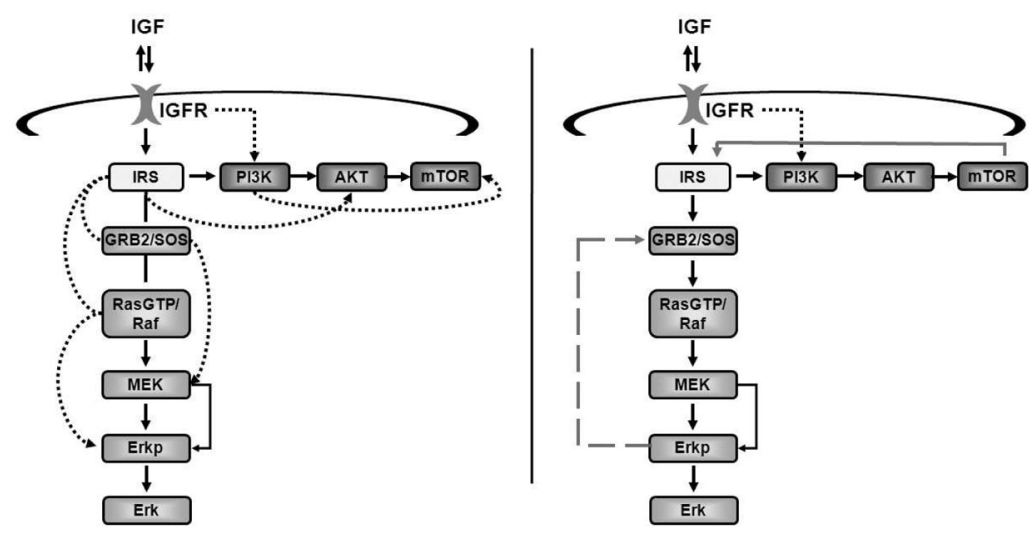

Figure 3.

$\mathrm{BN}$ and GBN results from synthetic data, using one perturbation per sample. BN results are shown on the left, GBN results on the right. Broken arrows are noncausal. Missed edges are not indicated; the BN model misses 4 edges and contains 7 extra edges, the GBN model misses 2 edges and shifts the Erk $\rightarrow$ Grb2Sos edge as before. It also contains one noncausal edge. 

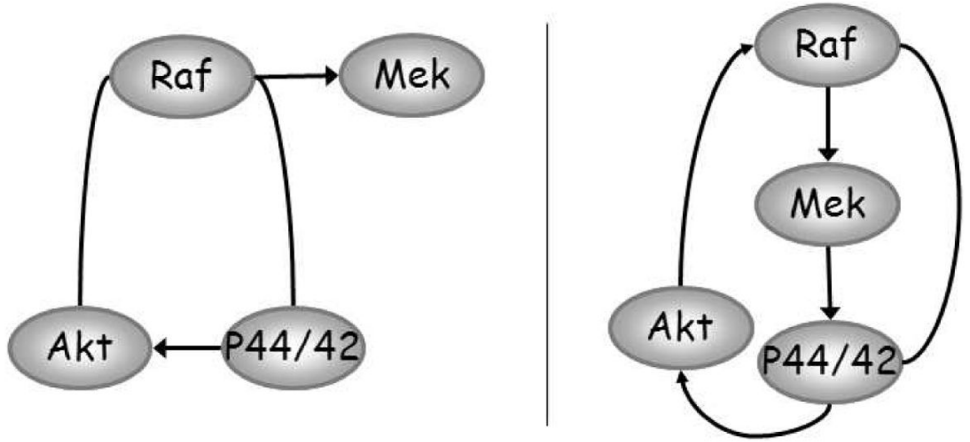

Figure 4.

$\mathrm{BN}$ and GBN results from real flow cytometry data, using one perturbation per sample. BN results are shown on the left, GBN results on the right. The $\mathrm{BN}$ model misses 1 edge and fails to orient 2 edges, the GBN model misses no edges but fails to orient one edge. 